\title{
MESENCHYMAL STEM CELLS PRODUCE FUNCTIONAL CARTILAGE MATRIX IN THREE-DIMENSIONAL CULTURE IN REGIONS OF OPTIMAL NUTRIENT SUPPLY
}

\author{
Megan J. Farrel1 ${ }^{1,2}$, Eric S. Comeau ${ }^{1,2}$ and Robert L. Mauck ${ }^{1,2 *}$ \\ ${ }^{1}$ Department of Orthopaedic Surgery, McKay Orthopaedic Research Laboratory, Perelman School of Medicine, \\ University of Pennsylvania, Philadelphia, PA, USA \\ ${ }^{2}$ Department of Bioengineering, School of Engineering and Applied Science, University of Pennsylvania, \\ Philadelphia, PA, USA
}

\begin{abstract}
Mesenchymal stem cells (MSCs) are a promising cell source for the treatment of musculoskeletal disease. However, MSC chondrogenesis in 3D culture generates constructs whose macroscopic (bulk) mechanical properties are inferior to constructs formed with chondrocytes. To investigate where and why these deficits in functionality arise, we assessed the local (microscopic) properties of cell-laden hydrogel constructs. Both chondrocyte- and MSC-laden constructs showed pronounced depth dependency, with $\sim 3.5$ and $\sim 11.5$ fold decreases in modulus from the surface to central regions, respectively. Importantly, in the surface region, properties were similar, suggesting that MSCs can produce matrix of mechanical equivalence to chondrocytes, but only in conditions of maximal nutrient support. Dynamic culture on an orbital shaker (which enhances diffusion) attenuated depth-dependent disparities in mechanics and improved the bulk properties compared to free swelling conditions (225 to $438 \mathrm{kPa}$ for chondrocytes, 122 to $362 \mathrm{kPa}$ for MSCs). However, properties in MSC-based constructs remained significantly lower due to persistent mechanical deficits in central regions. MSC viability in these central regions decreased markedly, with these changes apparent as early as day 21 , while chondrocyte viability remained high. These findings suggest that, under optimal nutrient conditions, MSCs can undergo chondrogenesis and form functional tissue on par with that of the native tissue cell type. However, the lack of viability and matrix production in central regions suggests that chondrogenic MSCs do not yet fully recapitulate the advanced phenotype of the chondrocyte, a cell that is optimized to survive (and thrive) in a mechanically challenging and nutrient-poor environment.
\end{abstract}

Keywords: Mesenchymal stem cells; chondrogenesis; three-dimensional culture; cartilage; mechanical properties

\author{
*Address for correspondence: \\ Robert L. Mauck, PhD \\ McKay Orthopaedic Research Laboratory \\ University of Pennsylvania \\ 424 Stemmler Hall \\ $36^{\text {th }}$ Street and Hamilton Walk \\ Philadelphia, PA 19104, USA
}

Telephone Number: 1-215-898-3294

FAX Number: 1-215-573-2133

E-mail: lemauck@mail.med.upenn.edu

\section{Introduction}

Mesenchymal stem cells (MSCs) were first described in the 1970s as an adherent, colony forming fibroblastlike cell population within bone marrow (Friedenstein et al., 1970). Since that time, numerous studies have investigated their capacity to undergo self-renewal and multipotential differentiation. Together, these studies have demonstrated that MSCs are readily expandable in in vitro culture and can undergo adipogenesis, osteogenesis, and chondrogenesis when provided the appropriate soluble factors and culture environments (Johnstone et al., 1998; Pittenger et al., 1999). As MSCs can be isolated from adults, they might serve as an autologous cell type for regenerative medicine applications to treat tissue damage or disease, and are therefore an attractive cell source for the clinical treatment of chronic, non-healing musculoskeletal diseases.

Articular cartilage is one key target for stem cell therapy in the musculoskeletal system. This dense white connective tissue lines the surfaces of all major diarthrodial joints (hips, knees, etc) and functions to transmit and distribute load (Ateshian and Hung, 2005). However, the tissue is avascular in the adult, and as a consequence, has a limited nutrient supply and poor intrinsic healing capacity. Short of total joint replacement, current clinical practices for cartilage repair include microfracture (broaching the subchondral bone interface to allow stem cells to access the wound site from the marrow) or delivery of exogenously expanded autologous chondrocytes (autologous chondrocyte implantation; ACI) (Vijayan et al., 2010). In both cases, limited defect size, fragile fibrocartilaginous repair tissue and long recovery periods reduce efficacy and widespread application (LaPrade et al., 2008; Nehrer et al., 1999; Van Assche et al., 2010).

To improve on these therapies, tissue engineering and regenerative medicine approaches have been developed that combine three-dimensional (3D) scaffold materials, cells, and soluble factors to generate cartilage-like materials. Engineered tissues rich in type II collagen and aggrecan (markers of mature cartilage) with mechanical properties comparable to native tissue have been fabricated from a number of starting biomaterials (Chung and Burdick, 2008). Improvements in culture methods, including tailored biochemical and mechanical stimulation, have further improved the in vitro development of these constructs (Hung et al., 2004). Indeed, recent studies have shown that chondrocytes encapsulated in agarose can produce cartilage-like materials with near-native 
mechanical properties (Byers et al., 2008; Lima et al., 2007). Despite this progress, limitations in the use of chondrocytes include the requirement of invasive harvest from non-diseased, non-load bearing sites within the joint, as well as the limited activity and health of these cells when derived from adults. Therefore, interest has focused on the use of adult-derived MSCs for cartilage tissue engineering applications.

As with chondrocytes, steady improvements in chondrogenic growth conditions, three-dimensional scaffold design, and mechanical loading regimens have significantly enhanced construct formation using MSCs (Huang et al., 2010b). Like chondrocytes, these cells can be readily encapsulated and differentiate in a number of different 3D systems (Huang et al., 2010b). However, limitations in MSC potential become apparent with long term culture in these 3D contexts. Namely, when cultured identically, MSCs produce matrix of a lower modulus when compared to chondrocytes (Erickson et al., 2009; Huang et al., 2010a; Huang et al., 2009; Lima et al., 2007; Mauck et al., 2006). On a molecular level, direct comparisons between differentiated MSCs and chondrocytes revealed many hundreds of genes that remain differentially regulated between the two cell types (Boeuf et al., 2008; Huang et al., 2010c). Likewise, while mechanical pre-conditioning has been shown to improve the mechanical properties of MSC-based constructs (Huang et al., 2010a; Meyer et al., 2011), these improvements are small in comparison to the same stimuli applied to chondrocyte-based constructs (Lima et al., 2007).

Together, these data suggest that, on a bulk level, MSCs do not fully replicate the properties or potential of native tissue chondrocytes. However, a significant limitation of this previous work was the fact that all analyses were performed on whole constructs that perforce contain a potentially heterogeneous population of cells (Halleux et al., 2001; Mareddy et al., 2007; Okamoto et al., 2002; Pittenger et al., 1999; Russell et al., 2010) and are of sufficient size as to allow for the development of diffusional gradients across the construct expanse (Buckley et al., 2012). In such conditions, nutrient and growth factor utilization at the periphery may limit MSC differentiation and matrix production away from these sources. Bulk analysis of molecular expression and mechanical properties would therefore blur any variations that arise from these gradients, and so fail to identify differential chondrogenic efficacy as a function of the changing microenvironment. Furthermore, oxygen consumption by MSCs in chondrogenic pellet culture is nearly 10 fold higher than that of freshly isolated chondrocytes (i.e., $12.3 \mathrm{fmol} / \mathrm{h} / \mathrm{cell}$ (Pattappa et al., 2011) vs. 1.34 $\mathrm{fmol} / \mathrm{h} / \mathrm{cell}$ (Heywood and Lee, 2008)). This differential utilization of metabolites (due to persistent differences in cell metabolism) would exacerbate nutritional gradients throughout the construct, and would likely impact the development of functional properties in regions away from the construct periphery.

To address these issues, this study sought to determine whether the differences in macroscopic properties observed in MSC-based constructs result from inadequate chondrogenic induction throughout the construct or from spatially varying matrix production and properties. For this, we used fluorescence microscopy and digital image correlation to investigate the mechanical properties of matrix produced by MSCs and chondrocytes on a microscopic scale. Similar techniques have been used for the investigation of the depth-dependent mechanical properties of native cartilage (Schinagl et al., 1997; Wang et al., 2001) and tissue engineered chondrocyte-laden constructs (Kelly et al., 2006; Klein et al., 2007; Klein and Sah, 2007). Our objective was to identify where mechanical properties in MSC-laden constructs are lowest and to determine mechanistically why these differences arise relative to chondrocyte-based constructs. Based on histological staining patterns, we hypothesized that MSC-laden constructs would develop depth-dependent mechanical properties resultant of nutrient and waste gradients, compared to more homogeneous profiles for chondrocyte-laden constructs. Furthermore, we hypothesized that dynamic culture and improved solute transport would reduce the depth-dependency of MSCladen constructs and result in a significant increase in macroscopic mechanical properties compared to freeswelling conditions.

\section{Materials and Methods}

\section{Cell isolation, 3D encapsulation, and culture}

Unless otherwise stated, reagents were purchased from Sigma-Aldrich (St. Louis, MO, USA). Juvenile bovine MSCs were isolated from marrow from the femur and tibia of three donor calves (Research 87, Boylson, MA, USA) (Mauck et al., 2006). MSCs were expanded through passage 2 in medium consisting of high glucose Dulbecco's Modified Eagle Medium (DMEM; Gibco, Invitrogen Life Sciences, Carlsbad, CA, USA), $10 \%$ fetal bovine serum (FBS, Gibco), and $1 \%$ Antibiotic-Antimycotic (PSF; Gibco). Donor-matched primary chondrocytes were isolated from the carpometacarpal cartilage of the three donors. Diced cartilage was subjected to pronase digestion (0.25 mg/mL, $1 \mathrm{~h}, 37^{\circ} \mathrm{C}$; Calbiochem/EMD Chemicals, Gibbstown, NJ, USA) followed by collagenase digestion $\left(0.5 \mathrm{mg} / \mathrm{mL}, 6 \mathrm{~h}, 37{ }^{\circ} \mathrm{C}\right)$ (Mauck et al., 2003). After expansion (MSCs) or digestion (chondrocytes), cells were encapsulated in $2 \%$ agarose. Briefly, cells were suspended in a chemically defined media (CM) at a density of 40 million cells $/ \mathrm{mL}$. The cell suspension was then mixed with molten $4 \% \mathrm{w} / \mathrm{v}$ agarose (type VII, $49^{\circ} \mathrm{C}$ ) in phosphatebuffered saline (PBS) at a 1:1 ratio and cast between two parallel plates separated by $2.25 \mathrm{~mm}$ spacers (Mauck et al., 2003; Mauck et al., 2006). Using a biopsy punch, $2 \%$ agarose constructs ( $4 \mathrm{~mm}$ in diameter, $2.25 \mathrm{~mm}$ in depth) were extracted with an initial cell density of 20 million cells/mL. CM consisted of high glucose DMEM, $1 \%$ PSF, $0.1 \mu \mathrm{M}$ dexamethasone, $50 \mu \mathrm{g} / \mathrm{mL}$ ascorbate 2-phospate, $40 \mu \mathrm{g} / \mathrm{mL}$ insulin, $6.25 \mu \mathrm{g} / \mathrm{mL}$ transferrin, $6.25 \mathrm{ng} / \mathrm{mL}$ selenous acid, $1.25 \mathrm{mg} / \mathrm{mL}$ bovine serum albumin, and $5.35 \mu \mathrm{g} / \mathrm{mL}$ linoleic acid. 


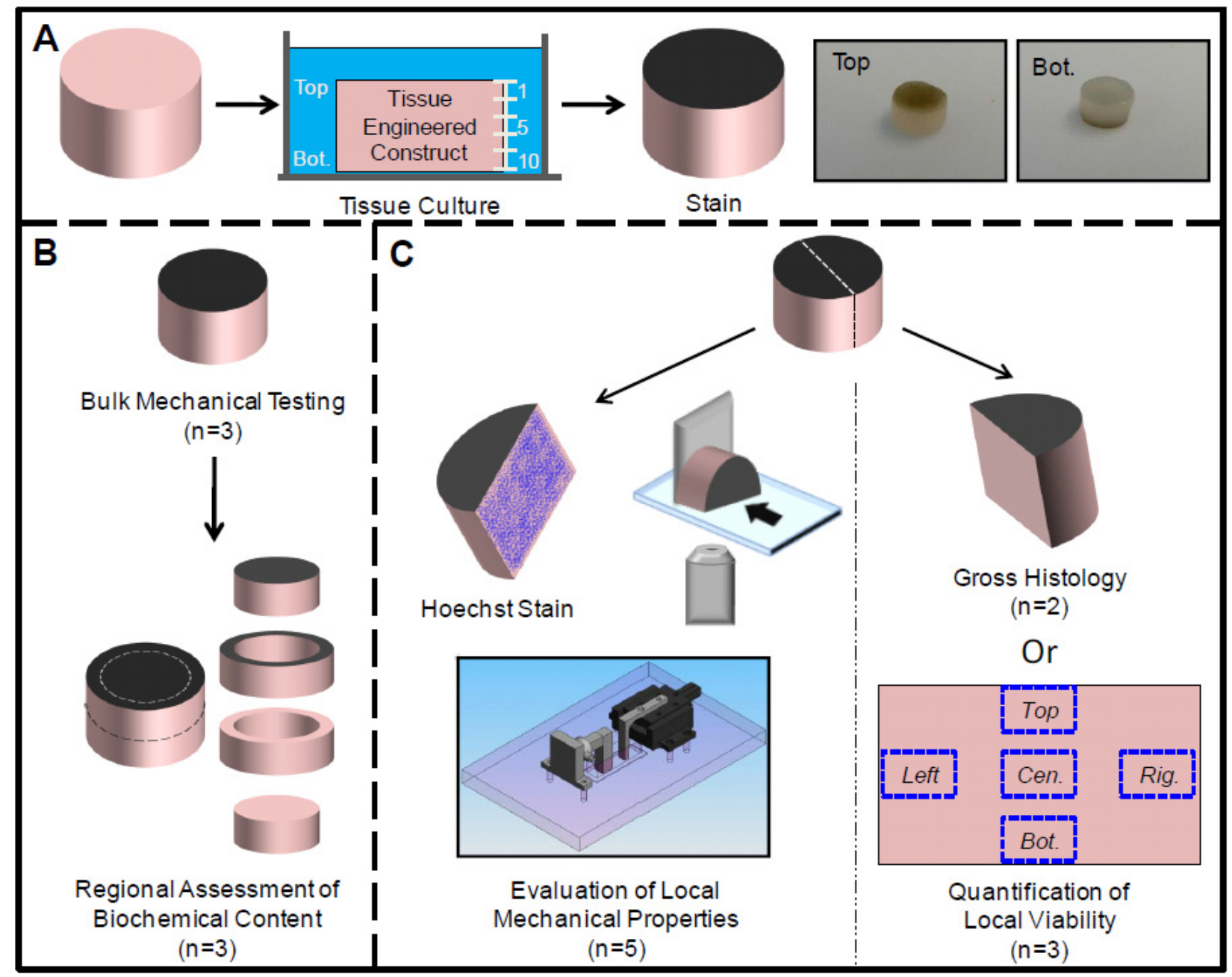

Fig. 1. Study design schematic. (A) Construct orientation (4 mm diameter, $2.25 \mathrm{~mm}$ thick) was maintained throughout the culture period. Construct tops (Region 1) were stained after removal from the tissue culture well plate to maintain orientation through mechanical testing. (B) Following bulk testing, constructs were cored and halved through the transverse plane for biochemical assessment. (C) Prior to local mechanical property assessment, constructs were halved through the median plane. Half of the construct was stained and tested in a custom microscope-based uniaxial compression device. The remaining half was preserved for histology or regional viability assessment.

\section{Construct culture}

Constructs were cultured under free swelling or dynamic conditions over 9 weeks. For free swelling conditions, constructs were cultured in CM with (FS (+TGF- $\beta 3)$ ) or without (FS (-TGF- $\beta 3)$ ) the addition of $10 \mathrm{ng} / \mathrm{mL}$ transforming growth factor-beta 3 (TGF- $\beta 3$; R\&D Systems, Minneapolis, MN, USA). Dynamically cultured constructs were cultured in CM with TGF- $\beta 3$ (Dyn (+TGF- $\beta 3$ )) while being subjected to continuous orbital shaking at $1.2 \mathrm{~Hz}(115 \mathrm{~V}-25$ x 25 Orbital Shaker, BellCo Glass Inc, Vineland, NJ, USA). These conditions were chosen to provide continuous agitation of the medium, while ensuring that constructs did not tumble. For all culture conditions, care was taken to ensure constructs did not flip during handling and feeding. Constructs were fed twice weekly with $1 \mathrm{~mL}$ of medium per construct. Immediately upon removal from the well plate, the top surface of each construct was stained with a solution consisting of $50 \%$ $\mathrm{v} / \mathrm{v}$ PBS, $25 \% \mathrm{v} / \mathrm{v}$ hematoxylin solution $(2.5 \% \mathrm{w} / \mathrm{v}$ hematoxylin (Thermo Fisher Scientific, Hampton, New
Hampshire) in $95 \%$ ethanol), $12.5 \% \mathrm{v} / \mathrm{v}$ aqueous ferric chloride $(10 \% \mathrm{w} / \mathrm{v}$ ferric chloride (Fisher) in distilled water), and $12.5 \%$ Wiegert's iodine (Fisher) to maintain orientation throughout testing (Fig. 1A).

\section{Mechanical analysis of bulk properties}

Constructs $(n=3)$ were tested in uniaxial unconfined compression for the assessment of bulk properties as in (Mauck et al., 2000). Constructs were first equilibrated under a $0.02 \mathrm{~N}$ static load for $5 \mathrm{~min}$, followed by evaluation of equilibrium stress ( $1000 \mathrm{~s}$ stress relaxation) following application of $10 \%$ strain (at a rate of $0.05 \% / \mathrm{s}$ ). Equilibrium modulus was calculated from the equilibrium stress and sample geometry. Following mechanical testing, constructs were cored with a $3 \mathrm{~mm}$ biopsy punch and bisected through the transverse plane, resulting in 4 sections of roughly equal volume including: top annulus, top core, bottom annulus, and bottom core. Construct regions were frozen separately at $-20{ }^{\circ} \mathrm{C}$ for regional assessment of biochemical content (Fig. 1B). 


\section{Mechanical analysis of local properties}

Constructs $(n=5)$ were halved diametrically for local assessment of compressive strain via digital image correlation. The construct half was stained with $80 \mu \mathrm{g} / \mathrm{mL}$ Hoechst 33342 (Molecular Probes, Invitrogen) to label cell nuclei as fiducial markers; the remaining half was reserved for histology $(n=2)$ or assessment of viability $(n=3)$. Stained construct halves were placed in PBS in a custom unconfined compression tester (modified after Knight et al. (1998)) situated on the stage of a Nikon Eclipse TE2000-U inverted microscope (Nikon Instruments Inc, Melville, NY, USA). Uniaxial compression was applied in $4 \%$ platen-to-platen strain increments through $12 \%$ strain, with a 7 min relaxation period following each compressive step (Fig. 1C). Images were acquired at $0 \%$ strain and at equilibrium for each strain increment with 3x magnification. Load at equilibrium was recorded for each increment. Sequential images were analyzed using Vic2D (Correlated Solutions, Columbia, SC, USA), and Lagrangian strain $\left(\mathrm{E}_{\mathrm{xx}}\right)$ was calculated with $\mathrm{x}$ defined as the direction of loading. Lagrangian strain values, at $12 \%$ platen-to-platen engineering strain, were binned into ten regions of equal size through the depth of the construct using a custom MATLAB script (The MathWorks Inc, Natick, MA, USA) and averaged to obtain average strain values through the depth. These values, coupled with the equilibrium boundary stresses, were used to calculate local modulus through the depth. Region of analysis was restricted to the inner $80 \%$ of the construct (Region 2 to Region 9) due to edge effects of the testing modality.

\section{Histological analysis}

Construct halves $(n=2)$ were fixed in $4 \%$ paraformaldehyde (FD NeuroTechnologies Inc, Ellicott City, MD, USA), dehydrated with a series of ethanol washes, and paraffin embedded (Fig. 1C). Sections $(8 \mu \mathrm{m})$ were rehydrated and stained for proteoglycans (Alcian Blue, Rowley Biochemical Inc, Danvers, MA, USA). Additional sections were rehydrated, incubated in hyaluronidase $(1 \mathrm{mg} / \mathrm{mL})$ for $2 \mathrm{~h}$ at $37^{\circ} \mathrm{C}$ to remove proteoglycans, and stained for collagens (Picrosirius Red) as in Melrose et al. (2004). Stain intensity through the depth of the construct was assessed using the plot profile function of Image J (NIH). Additional sections were stained for apoptotic markers using the FragEL DNA Fragmentation Detection Kit (Calbiotech, Spring Valley, CA, USA) according to manufacturer's instructions. Cells positive for apoptosis were indicated by co-localization of DAPI and TUNEL stains.

\section{Biochemical analysis}

Samples were papain digested as in Mauck et al. (2006) at $60{ }^{\circ} \mathrm{C}$ for $24 \mathrm{~h}$. The supernatant was assessed for sulfated glycosaminoglycan (GAG) content with the 1,9-dimethylmethylene blue dye-binding assay (Farndale et al., 1986) and collagen content with the orthohydroxyproline assay (Stegemann and Stalder, 1967). GAG and collagen content is presented as percent of construct wet weight.

\section{Quantification of viability}

Construct halves $(n=3)$ were stained with the LIVE/ DEAD Cell Viability Assay Kit for mammalian cells (Molecular Probes, Invitrogen, Life Sciences) for $30 \mathrm{~min}$ in PBS. Stained construct halves were imaged under 10x magnification, with calcein and ethidium-homodimer-1 signal acquired in the same focal plane in 5 regions of the bisected face including: center, top, bottom, left, and right (Fig. 1C). Constructs were aligned and centered under $4 \mathrm{x}$ magnification to ensure consistency of regional assessment. A custom MATLAB (The MathWorks, Inc.) script was used to automate counting of cells in each image. Local percent viability was calculated in each region, as well as aggregate viability as the percent ratio of live cells to the total number of cells within all five regions. Total cell count per area was recorded to ensure any change in percent viability was the result of cell death rather than an increase in cell number.

\section{Statistical analysis}

The statistical software SYSTAT (Systat Software, Inc., Chicago, IL, USA) was used to carry out ANOVA with Tukey's post-hoc testing to enable pairwise comparisons between groups. Data are presented as the mean and the standard deviation, with significance set at $p<0.05$. Threeway ANOVA was conducted for bulk equilibrium modulus, aggregate viability, aggregate cell count, and central cell count, with cell type, day, and culture condition as independent variables. Additional three-way ANOVA was conducted for local modulus, local cell count, and collagen content with cell type, region, and culture condition as independent variables. Four-way ANOVA was conducted for GAG content with cell type, region, culture condition, and day as independent variables. Two-way ANOVA was conducted for local modulus with cell type and culture condition as independent variables. One-way ANOVA was conducted for local strain and local day 63 viability, with region as the independent variable.

\section{Results}

\section{Bulk mechanical properties depend on culture conditions}

Consistent with previous findings for MSC- and chondrocyte-seeded constructs (Erickson et al., 2009; Huang et al., 2010a; Mauck et al., 2006), construct opacity (Fig. 2A) and equilibrium modulus increased with time for all free swelling groups (day $1 v s$. day 63, $p<0.001$ ), with the exception of MSC FS(-TGF- $\beta 3$ ) (Fig. 2B). Whereas chondrocyte-laden $\mathrm{FS}(+\mathrm{TGF}-\beta 3$ ) construct equilibrium modulus increased from day 42 to day $63(p=0.001)$, MSC-laden FS(+TGF- $\beta 3$ ) constructs plateaued over this same time period, reaching 129 and $122 \mathrm{kPa}$ on day 42 and day 63 , respectively. Conversely, when cultured with continual agitation (Dyn), the equilibrium modulus of day 63 MSC Dyn(+TGF- $\beta 3$ ) constructs increased compared to day $42(p<0.001)$ and was $\sim 3$-fold higher than day $63 \mathrm{MSC}$ $\mathrm{FS}(+\mathrm{TGF}-\beta 3)(p<0.001)$. Indeed, under these conditions, the equilibrium modulus of day 63 MSC Dyn(+TGF- $\beta 3$ ) constructs approached day 63 chondrocyte Dyn(+TGF- $\beta 3$ ) levels $(\sim 20 \%$ lower, $p<0.01)$. 


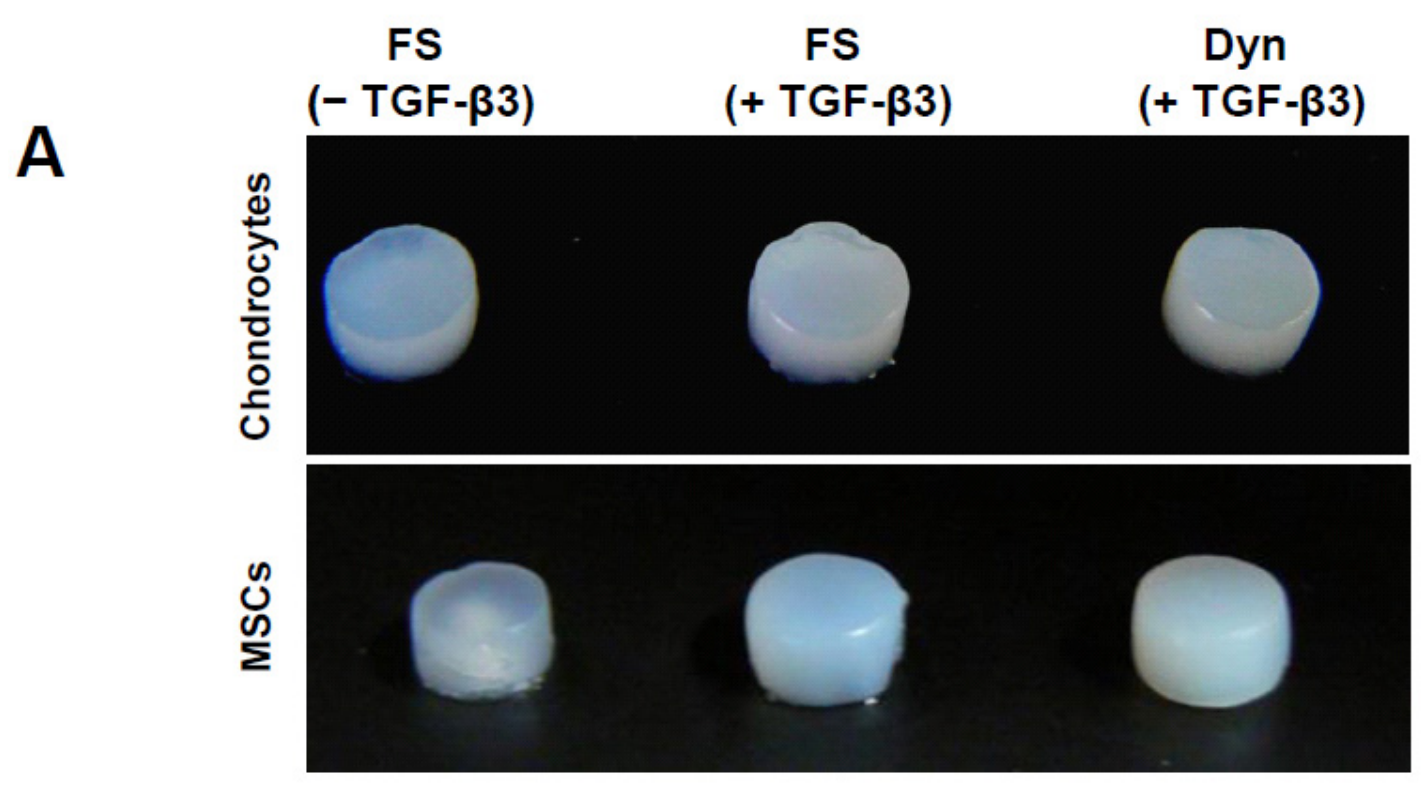

B

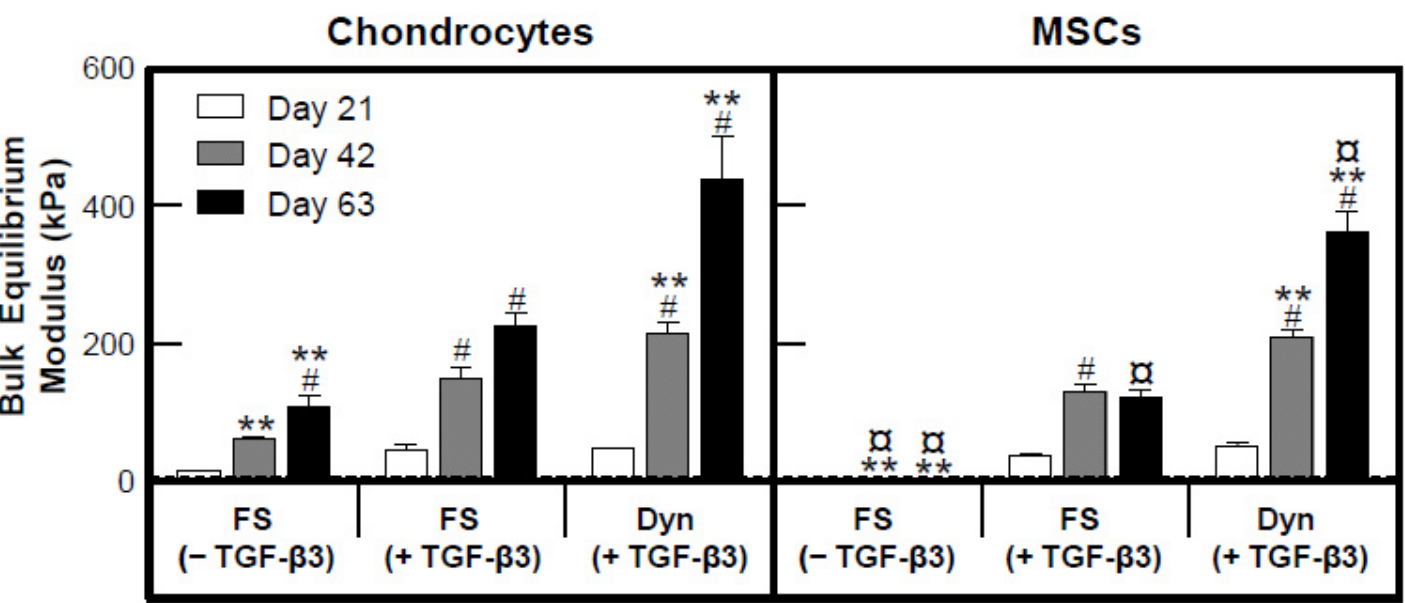

Fig. 2. Gross appearance and bulk assessment of unconfined compressive properties of constructs cultured in free swelling (FS) or orbital shaking (Dyn) conditions, with $(+)$ or without $(-)$ TGF- $\beta 3$. (A) Gross appearance of chondrocyte-laden (top) and MSC-laden (bottom) constructs on day 63. (B) Bulk construct equilibrium modulus was evaluated through day 63. Dotted lines denote day 1 equilibrium modulus values. (\# vs. all lower within culture condition and cell type; **vs. FS(+TGF $\beta-3)$ within day and cell type; $\alpha$ $v s$. CH cultured identically, $p<0.05) . n=3$.

\section{Depth-dependent local mechanical properties}

To ascertain the origin of the differences in bulk mechanical properties, we next analyzed the local strain profiles within constructs during compressive deformation (Fig. $3 \mathrm{~A})$. This analysis showed that in the absence of TGF- $\beta 3$ (FS(-TGF- $\beta 3$ )), both chondrocyte-laden constructs and MSC-laden constructs had uniform strain profiles with time in culture, with little variation from the superficial zone (Region 2) to the deep zone (Region 9) (Fig. 3B). However, when cultured in the presence of TGF- $\beta 3$ (FS(+TGF- $\beta 3)$ ), both free swelling chondrocyte-laden and MSC-laden constructs developed depth-dependent strain profiles by day 21 . In these constructs, an $\sim 2.5$-fold increase in compressive strain was observed comparing superficial regions (Region 2) to middle regions (Regions 5 and 6) for chondrocyte-laden constructs. For MSC-laden constructs, this difference was even more marked, with an $\sim 6$-fold increase in strain in the center of the construct compared to the top surface. Once established, these depth dependent profiles were consistent through day 63 for both cell types.

When cultured in dynamic conditions with TGF- $\beta 3$, a shift in strain profiles for both cell types was observed. For chondrocyte-laden constructs, the central regions of the construct deformed least. An $\sim 2$-fold increase in compressive strain from Regions 5 and 6 to Regions 2 and 9 at day 21 persisted through day 63 with a 2-fold increase in strain from Regions 5 and 6 to Region 9. Conversely, for MSC-laden constructs cultured in dynamic conditions, the central regions remained highest in compressive strain (Regions 5 and 6). Although Regions 2 and 9 were no 
A

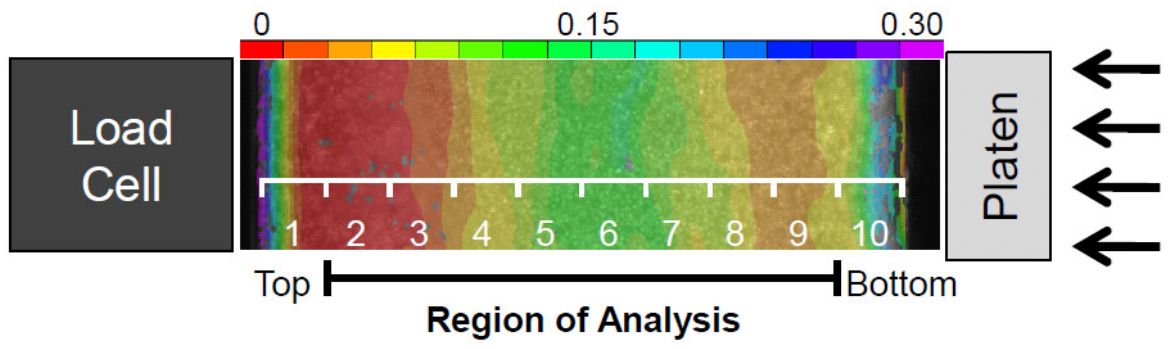

B

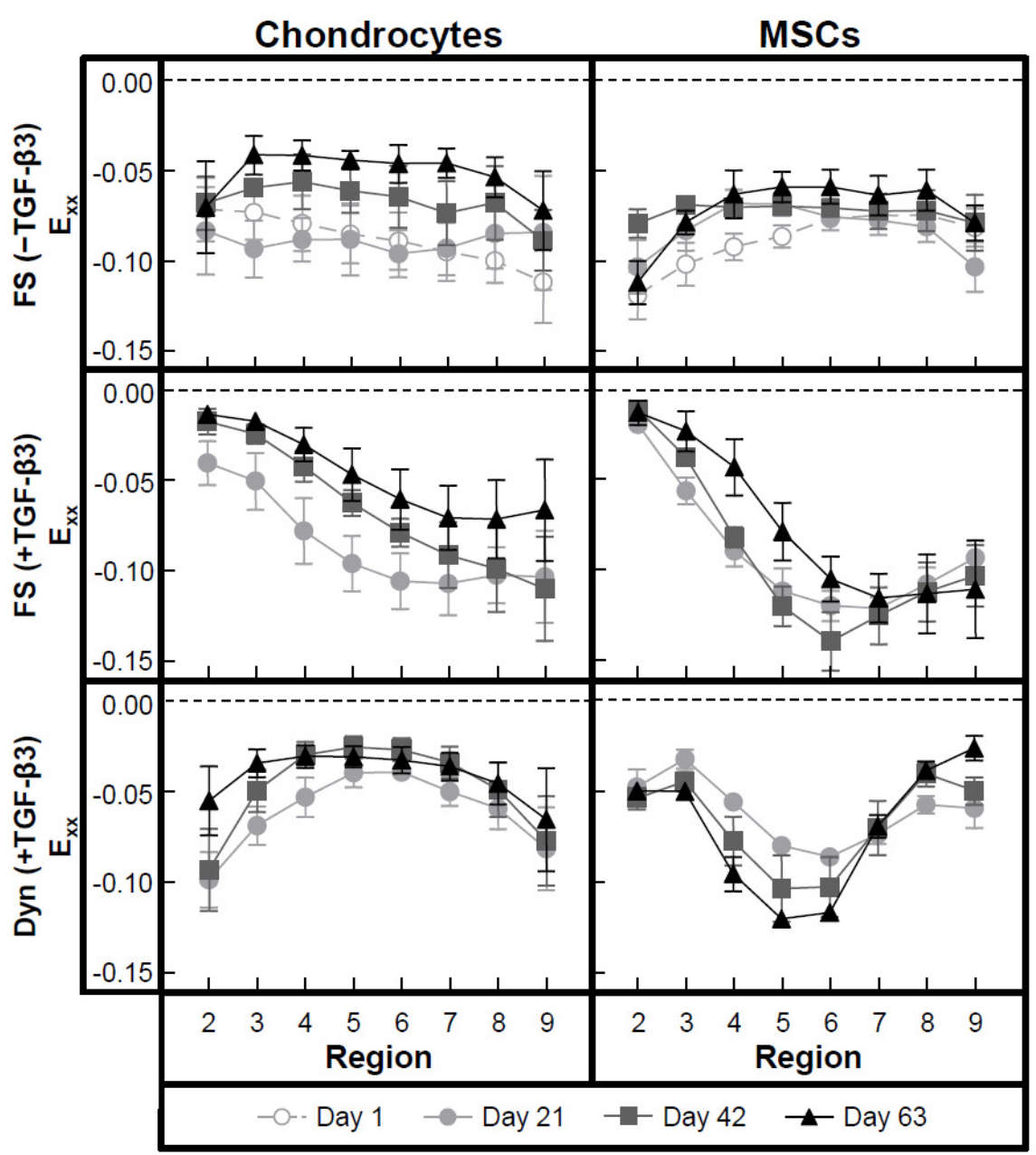

C

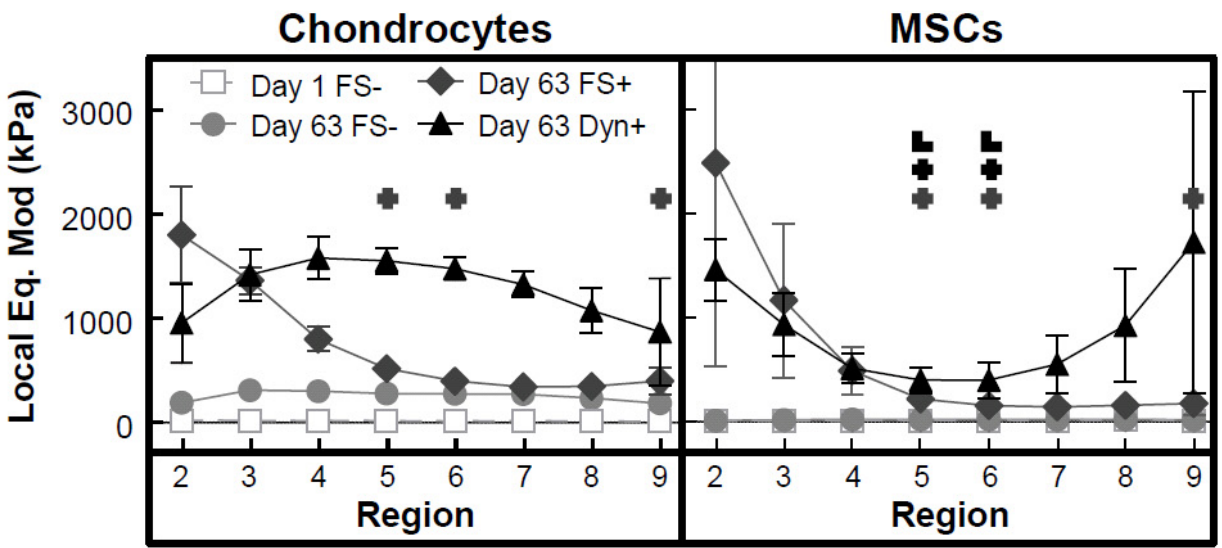

Fig. 3. Assessment of local compressive strain and equilibrium modulus. (A) Schematic of microscopic strain application and region of analysis with overlay of Vic $2 \mathrm{D} \mathrm{E}_{\mathrm{xx}}$ strain contour plot. (B) Compressive Lagrangian strain $\left(\mathrm{E}_{\mathrm{xx}}\right)$ through the depth of constructs as a function of time, cell type, and culture condition. Unique strain plot profiles developed as early as day 21 and persisted through day 63. (C) Day 63 local equilibrium modulus profiles as a function of cell type and culture condition. Dynamic culture reduced depth dependency in chondrocyte-laden constructs, but not for MSC-laden constructs, especially in the central regions. ( $v s$. Region 2; the Region 9, $p<0.05$ ). $n=5$. 


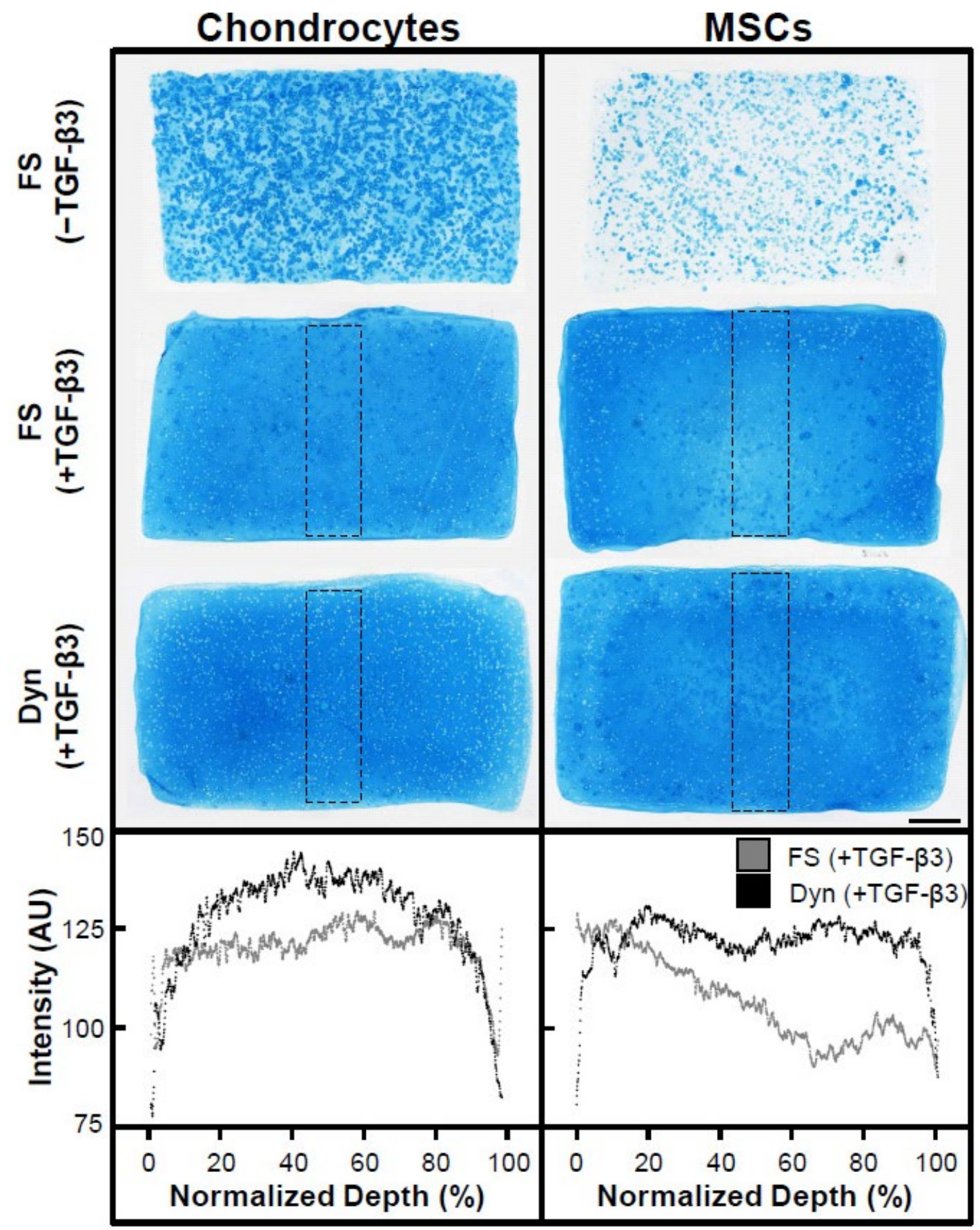

Fig. 4. (Top) Day 63 Alcian Blue staining of proteoglycans (PGs) as a function of cell type and culture condition. Dotted rectangle indicates area of intensity plot profiles. Scale bar $=500 \mu \mathrm{m}$. (Bottom) Stain intensity profiles of free swelling and dynamically cultured constructs in the presence of TGF- $\beta 3$. PG staining intensity mirrors local equilibrium modulus profiles with the exception of chondrocyte-laden FS(+TGF- $\beta 3$ ) constructs.

longer different for MSC-laden Dyn(+TGF- $\beta 3$ ) constructs at any time point, the central portion of the construct continued to show substantial deformation. MSC-laden Dyn(+TGF- $\beta 3$ ) constructs showed a 1.6-fold difference in center-to-edge strain at day 21 , which progressed to a 4.6-fold difference by day 63 (Region 9 vs. 5).

To better understand the implications depth-dependent strain profiles had on compressive properties, we calculated the local modulus though the depth on day 63 (Fig. 3C). Both chondrocyte and MSC-laden constructs cultured in the presence of TGF- $\beta 3$ in free swelling conditions
(FS(+TGF- $\beta 3)$ ) had a depth dependent modulus. In each case, the most superficial zone (Region 2) was stiffer than center and bottom regions $(5,6$, and $9, p<0.001)$. However, between cell types, the extent to which the local moduli values decreased was strikingly different. While there was an $\sim 3.5$-fold decline in modulus from Region 2 to Region 5 for chondrocyte-laden constructs, MSC-laden constructs showed an $\sim 11.5$-fold decrease in modulus. Furthermore, the lowest local modulus value for free swelling MSCladen constructs cultured with TGF- $\beta 3$ was $141 \mathrm{kPa}$, whereas the lowest value for chondrocyte-laden constructs 


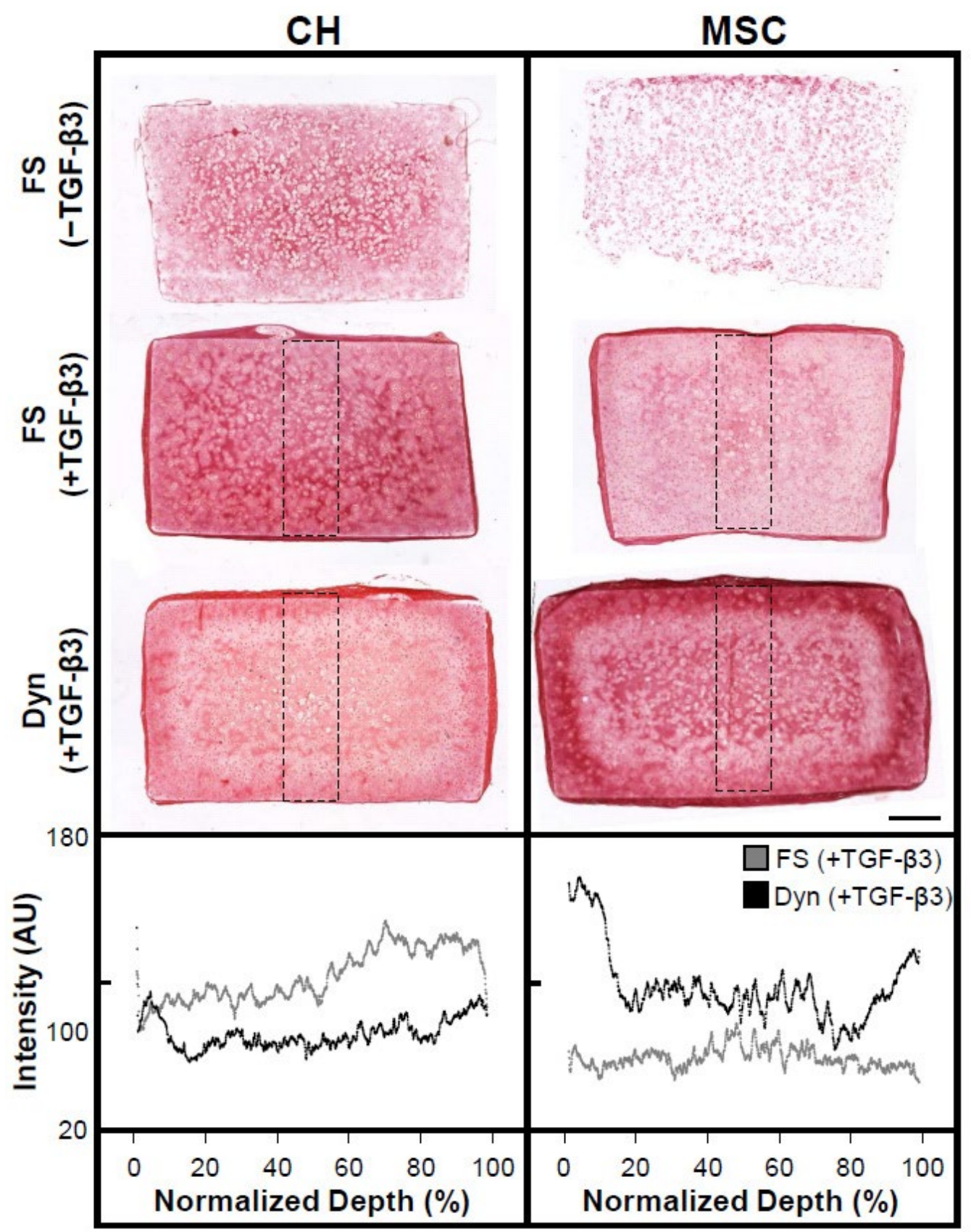

Fig. 5. (Top) Day 63 Picrosirius red staining of collagen as a function of cell type and culture condition. Dotted rectangle indicates area of intensity plot profiles. Scale bar $=500 \mu \mathrm{m}$. (Bottom) Stain intensity profiles of free swelling and dynamically cultured constructs in the presence of TGF- $\beta 3$.

was $341 \mathrm{kPa}$, both in Region 7. Of note, however, in the most superficial zone, MSC-laden and chondrocyte-laden constructs had moduli that were not different from one another $(p=0.877)$.

Dynamic culture resulted in a shift in this depth dependency, where the deepest region (Region 9) was no longer different from the most superficial region (Region 2) for MSC-laden constructs. However, under these dynamic conditions, the differences between MSC-laden constructs and chondrocyte-laden constructs within the central regions were further accentuated (Fig. 3C). In Regions 5 and 6, moduli for MSC-laden constructs increased compared to free swelling conditions (from 217 and $153 \mathrm{kPa}$ to 399 and $397 \mathrm{kPa}$ in Regions 5 and 6, respectively). However, central regions of chondrocyte-laden constructs remained significantly higher $(p<0.01)$ than MSC-laden constructs cultured identically, achieving 519 and $341 \mathrm{kPa}$ in free swelling conditions and 1553 and $1478 \mathrm{kPa}$ in dynamic culture conditions (Regions 5 and 6, respectively).

\section{Regional matrix distribution and content}

To determine the compositional basis of these depth dependent mechanical properties, the distribution of the principal cartilage extracellular matrix elements (i.e., proteoglycans and collagens) was assessed. Histological analysis showed that, after $63 \mathrm{~d}$ of culture, punctate pericellular accumulations of proteoglycans were present in both chondrocyte- and MSC-laden constructs in 


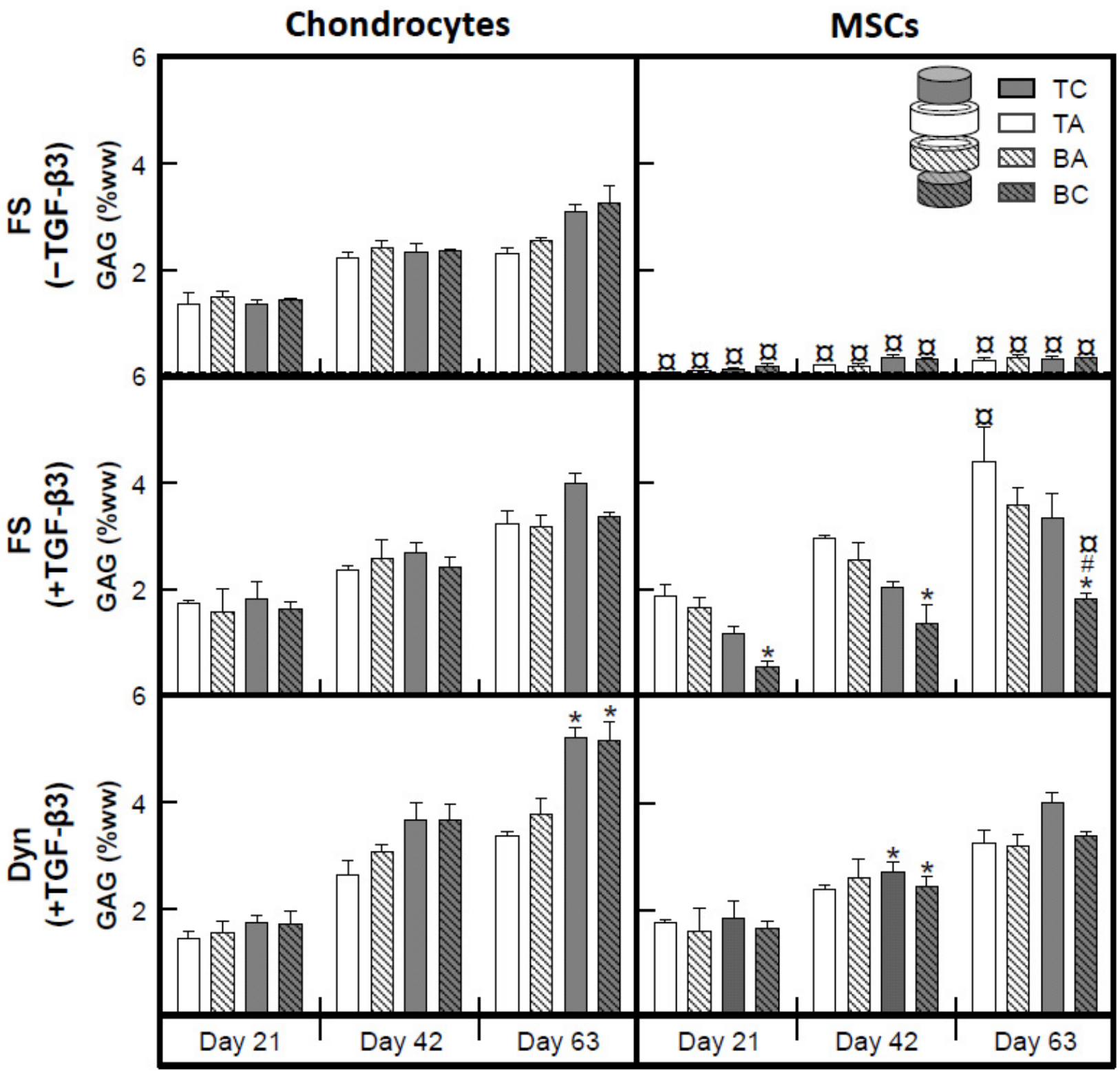

Fig. 6. Glycosaminoglycan (GAG) content (normalized by wet weight) as a function of region, time, cell type, and culture condition. Chondrocyte-laden constructs had a relatively homogenous GAG distribution. GAG content of MSC-laden constructs was highly dependent on region; GAG content regionality was relieved with dynamic culture. (\# vs. top of the same group; * vs. annulus of the same group; $\propto$ vs. chondrocyte of the same region cultured identically, $p<0.05) . n=3$.

FS(-TGF- 33 ) conditions, with less overall staining in the MSC-laden constructs (Fig. 4). There was a marked increase in overall staining intensity for MSC-laden $\mathrm{FS}(+\mathrm{TGF}-\beta 3$ ) constructs compared to constructs cultured without TGF- $\beta 3$. Quantification of staining intensity through the depth yielded a profile that mirrored that of the local equilibrium modulus, with the most intense staining near the top surface of the construct. Interestingly, depth dependence in staining intensity was not observed in chondrocyte-laden FS(+TGF- $\beta 3$ ) constructs, despite the measured depth-dependent mechanical profiles in these constructs. This finding may reflect limitations in the range over which Alcian blue staining can effectively discriminate between proteoglycan levels. Nevertheless,
Alcian blue staining intensity for both cell types in dynamic culture mirrored the measured mechanical profiles. Both MSC- and chondrocyte-laden Dyn(+TGF- $\beta 3$ ) constructs had the least intense staining right at the periphery of the constructs, indicating potential proteoglycan loss and/or dedifferentiation at this border. While chondrocyte-laden Dyn(+TGF- $\beta 3$ ) displayed the most intense staining in the central regions, the central-most regions of MSC-laden Dyn(+TGF- $\beta 3$ ) constructs had lower staining intensity compared to regions closer to the construct border. Similarly, inhomogeneous staining of collagens was observed, with the most intense staining occurring at the periphery of MSC-laden Dyn(+TGF- $\beta 3$ ) constructs (Fig. 5). Immunohistochemical staining of these sections (data 


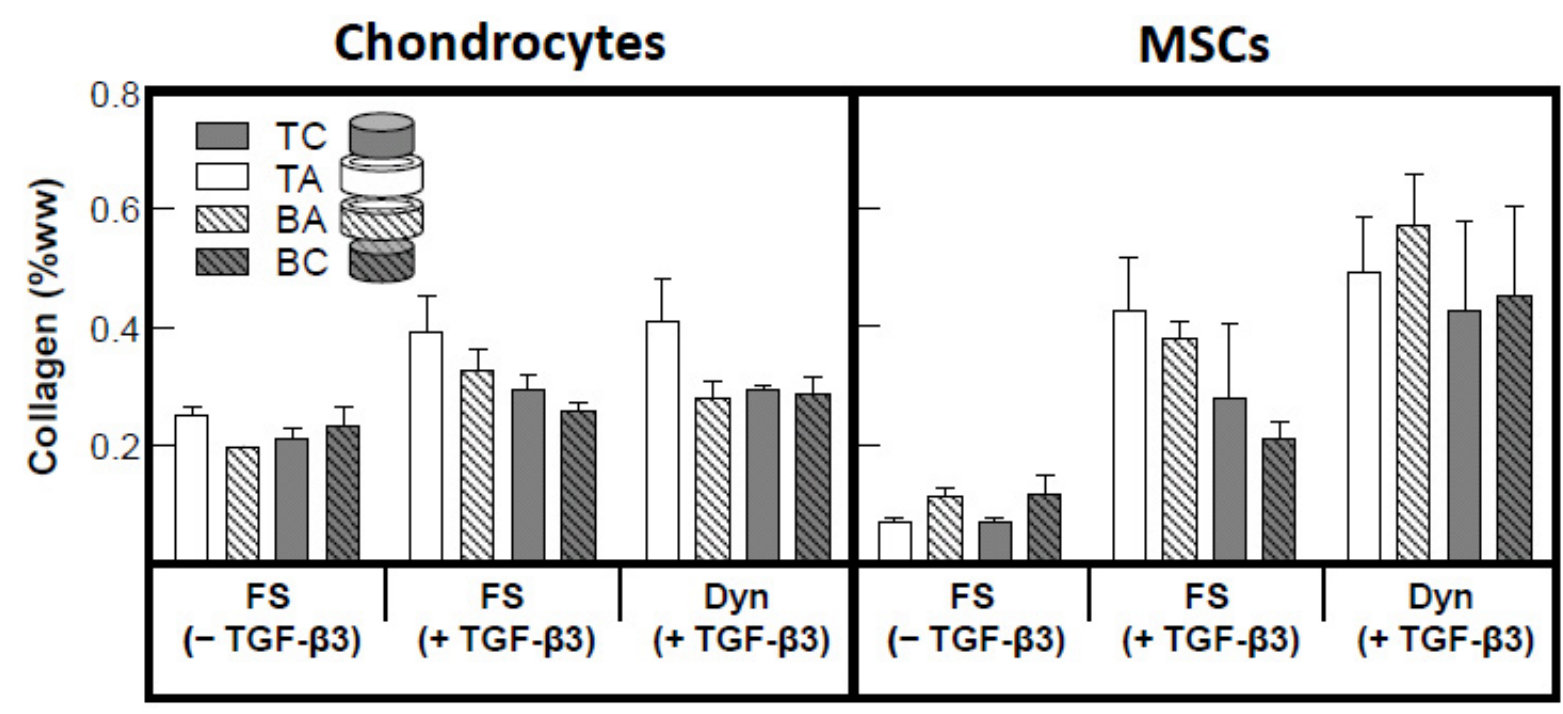

Fig. 7. Collagen quantification (normalized to wet weight) in day 63 constructs indicated low levels of collagen regardless of cell type, culture condition, and region. $n=3$.

not shown) revealed intense type II collagen staining and very low, cell-associated, type I collagen staining for all constructs cultured in the presence of TGF- $\beta 3$.

Regional quantification of glycosaminoglycan (GAG) and collagen content supported these histological findings. GAG content in chondrocyte-laden constructs was relatively uniform in the four regions of the construct assayed, regardless of culture condition and time, with the exception of chondrocyte-laden Dyn(+TGF- $\beta 3$ ) constructs, where GAG levels were $\sim 5.2 \%$ in the core regions, but only $\sim 3.5 \%$ in the annulus regions (Fig. 6). MSCs in free swelling culture without TGF- $\beta 3$ failed to produce appreciable amounts of GAG. Free swelling culture in the presence of TGF- $\beta 3$ resulted in inhomogeneous GAG production by day 63 , with the bottom core region of the construct having significantly lower GAG content $(1.8 \%)$ than the remaining three portions of the construct (top annulus $=4.4 \%$; top core $=3.3 \%$; bottom annulus $=3.6 \% ; p<0.05)$. Of note, in the top annulus region, FS(+TGF- $\beta 3$ ) MSC-laden constructs had significantly higher GAG content than this same region in chondrocyteladen constructs at day 63. Dynamic culture reduced this region dependency in GAG content in these MSC-laden constructs. Day 63 assessment of collagen content showed relatively low levels of collagen $(<1 \%)$ and little region dependency, regardless of cell type and culture condition (Fig. 7).

\section{Overall and regional chondrocyte and MSC viability}

To identify the underlying cellular mechanisms responsible for the establishment of these gradients in matrix deposition and depth-dependent mechanical properties, we next quantified cell viability as a function of time, location, cell type, and culture condition. Day 1 aggregate viability (the percent cell viability in all five regions) for chondrocyteladen and MSC-laden constructs was high (88\% and $82 \%$, respectively, Fig. 8A). With increased culture duration, viability in chondrocyte-laden constructs did not significantly change from day 1 values in any culture condition. Conversely, there were marked decreases in viability for all culture conditions in MSC-laden constructs. As early as day 21 , viability declined to $40 \%$ in FS(TGF- $\beta 3$ ) conditions, $41 \%$ in FS(+TGF- $\beta 3$ ) conditions, and $67 \%$ in Dyn(+TGF- $\beta 3$ ) conditions. Although viability in MSC-laden Dyn(+TGF- $\beta 3$ ) on day 21 was $\sim 1.5$-fold higher than FS(+TGF- $\beta 3$ ), these values declined with time such that differences were no longer significant by day 63 (Fig. 8A and B). Regional assessment of viability on day 63 showed a depth-dependent decline in viability in MSCladen FS(+TGF- $\beta 3$ ) constructs from the top surface to the central and bottom regions (Fig. 8C). Dynamic culture maintained an equivalent viability through the depth, though levels were markedly lower than day 1 in every region. TUNEL staining for apoptosis on day 21 (Fig. 9) revealed a low percentage of apoptotic chondrocytes within the center of the constructs, regardless of culture condition. Conversely, a marked increase in TUNEL-positive cells was observed in MSC-laden constructs under free swelling conditions. In Dyn(+TGF- $\beta 3$ ) conditions, fewer TUNELpositive MSCs were observed in the center of constructs.

\section{Discussion}

It is widely accepted that adult-derived MSCs hold promise for regenerative medicine and tissue engineering applications. Their utility has been proven in instances where the demands placed on the engineered system, whether mechanical or metabolic, are modest or supplemental in nature. For example, recent reports show that MSCs can successfully generate tissue-like mimics that reconstitute (or supplement) in vivo function (e.g., in the cartilaginous trachea (Macchiarini et al., 2008) or in myofibrous conduits (Dolgin, 2011)). However, these same cells fail to achieve functional parity with native tissue cells when more considerable functional demands 
A

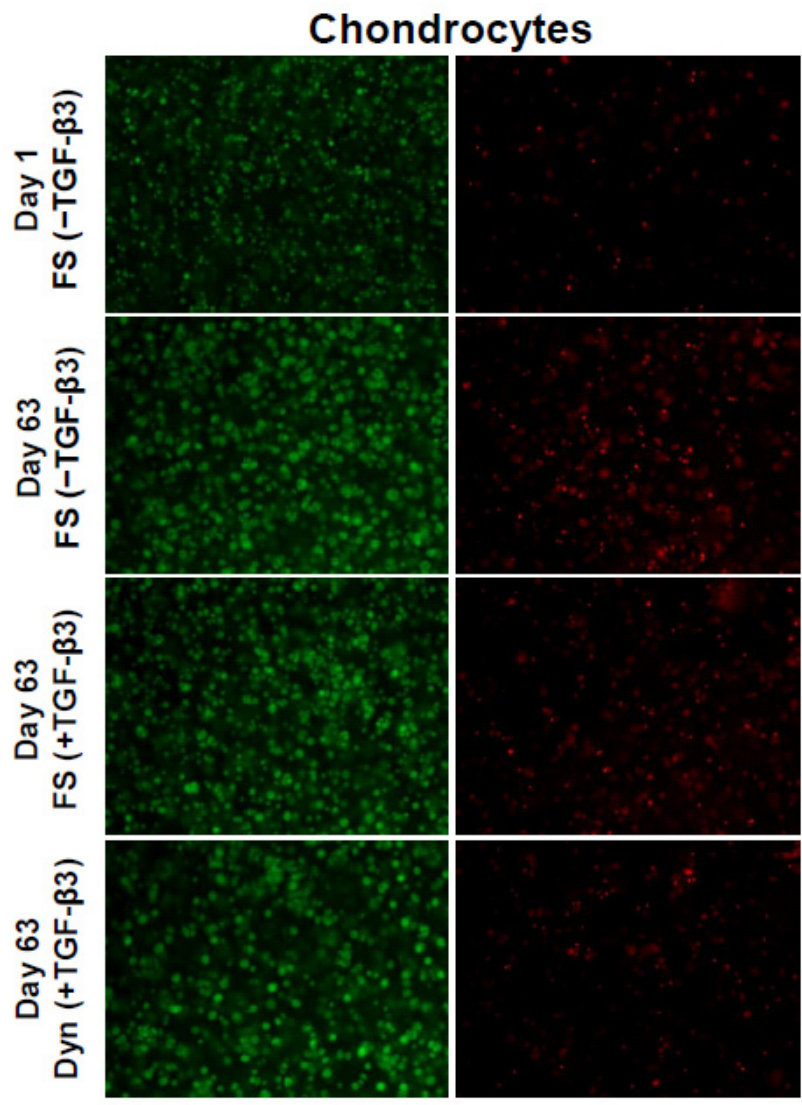

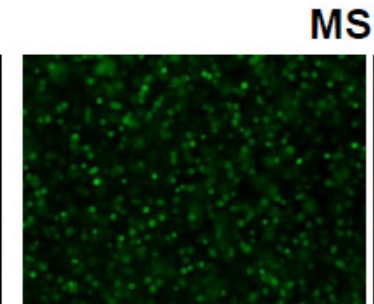

MSCs
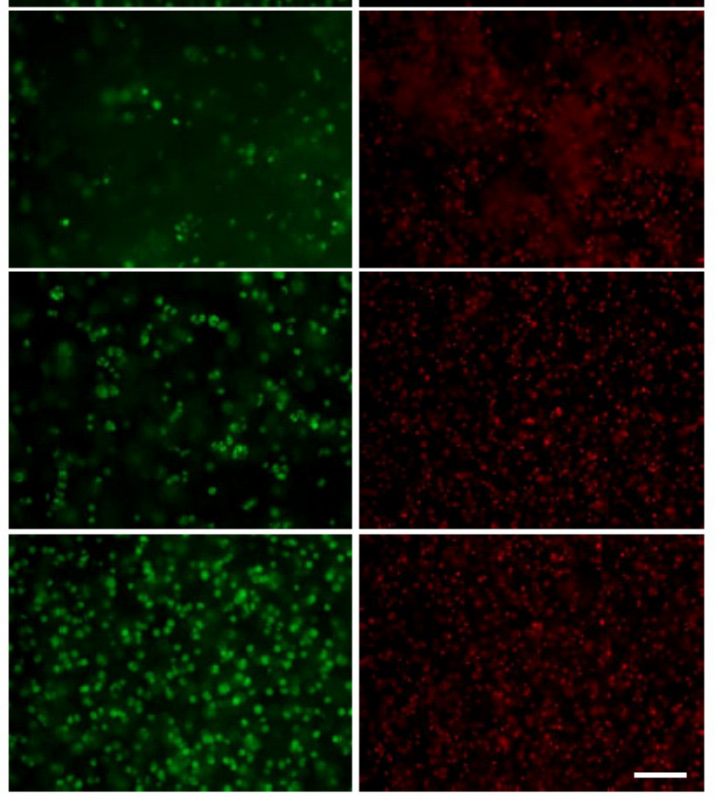

B

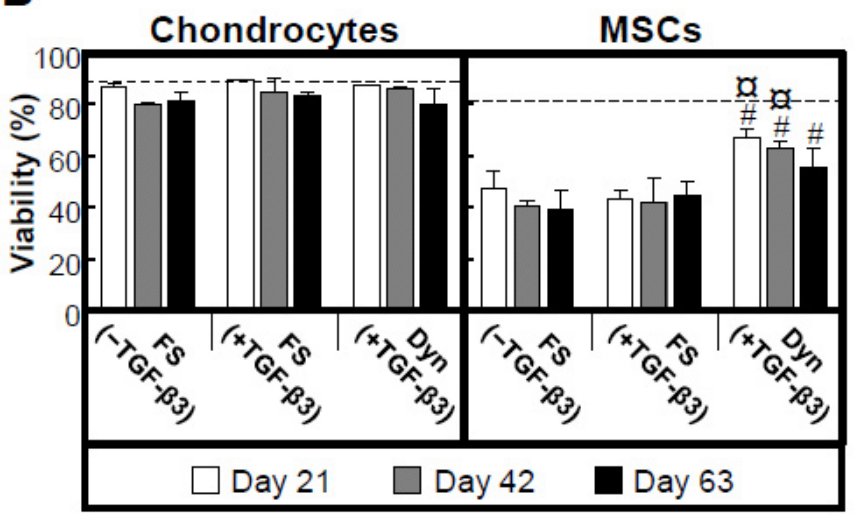

C

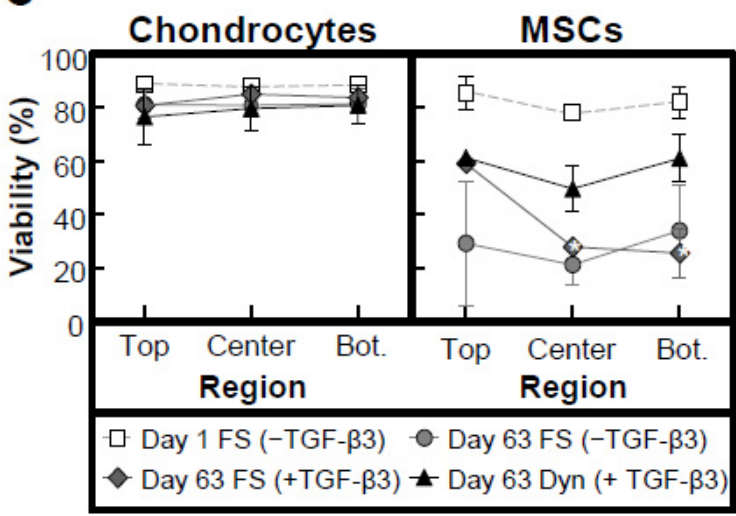

Fig. 8. Assessment of cell viability. (A) Central images of bisected constructs of both viable (green, left column) and dead (red, right column) cells. Scale bar $=100 \mu \mathrm{m}$. (B) Quantification of aggregate viability (from all five regions) as a function of time showed that chondrocyte viability remained relatively stable, while MSC viability declined significantly from day 1 values. Dotted line denotes mean day 1 viability. (\# vs. FS(-TGF- $\beta 3)$; $\alpha$ vs. FS(+TGF- $\beta 3$ ), $p<0.05$ ). (C) Analysis of viability through the depth of the constructs on day 63 revealed a significantly lower percentage of viable cells in the center and bottom regions of MSC FS(+TGF- $\beta 3$ ) constructs compared to the top region. (*vs. Top, $p<0.05) . n=3$.

are placed on the regenerate structure. For example, we and others have noted a striking deficiency in tissue engineered cartilage produced from MSCs relative to that produced by chondrocytes (Erickson et al., 2009; Huang et al., 2010a; Mauck et al., 2006), where the bulk mechanical properties of MSC-based constructs are $\sim 50 \%$ lower than that of chondrocyte-based constructs cultured identically. Moreover, on a molecular level (assessed across an entire construct), MSCs in 3D culture fail to fully establish the chondrogenic phenotype (Huang et al., 2010c), with the timing and magnitude of several hundred genes differentially regulated even after long periods of chondrogenic induction. This marked disparity in bulk expression likely contributes to the failure of these cells to produce a functional extracellular matrix.

A further complexity of these 3D culture systems is the spatially varying nutrient gradients that arise as a result of diffusional constraints and nutrient utilization at the construct boundaries. Such gradients in nutrient supply likely result in spatially and temporally varying 
FS(-TGF- $\beta 3)$
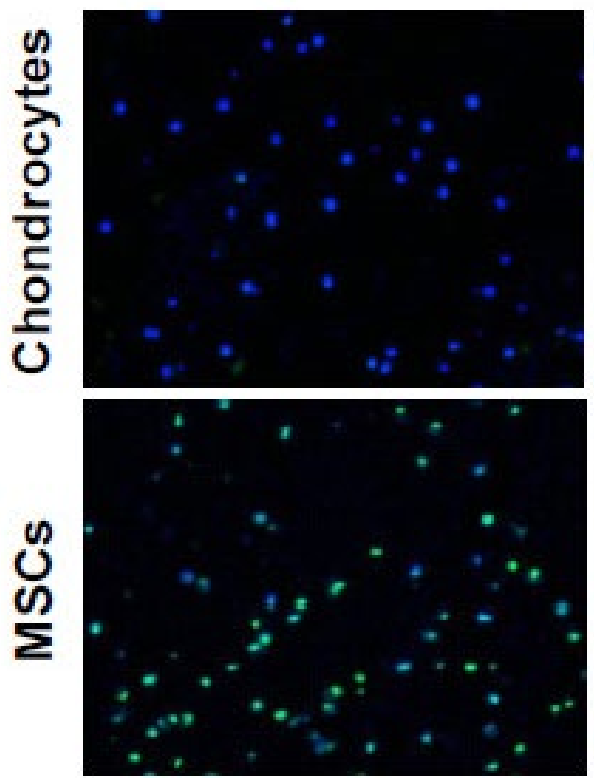

FS(+TGF- $\beta 3$ )
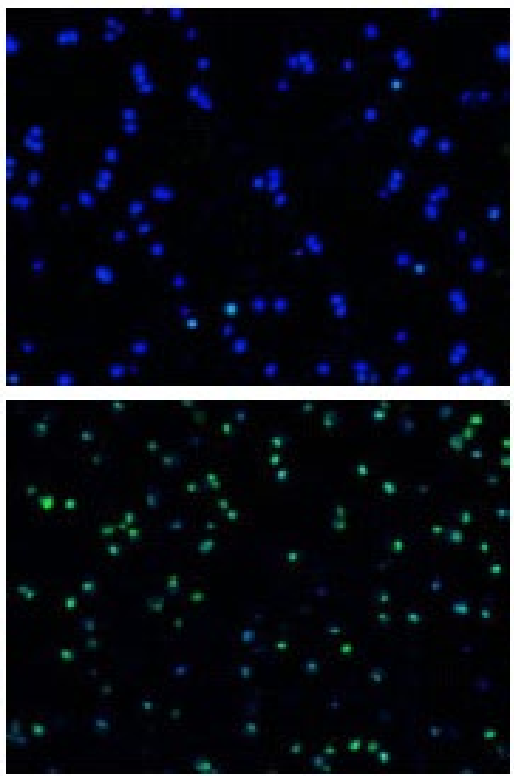

\section{Dyn(+TGF- $\beta 3)$}
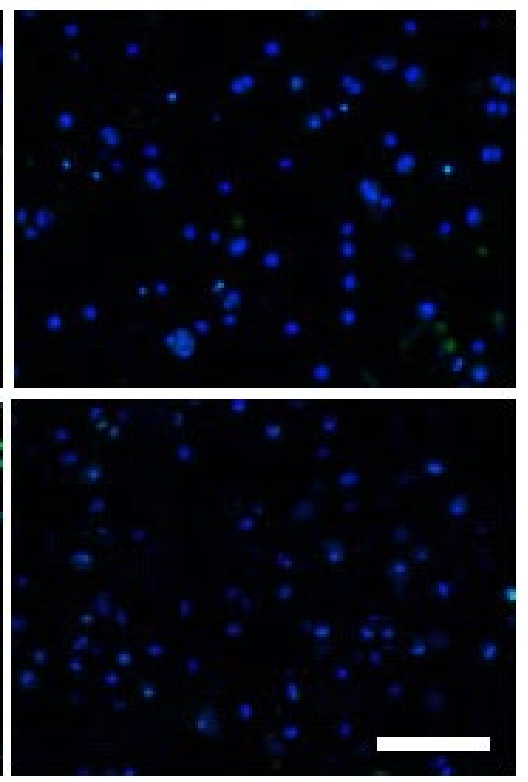

Fig. 9. TUNEL staining (green) in day 21 MSC-laden free swelling constructs (central region of the construct) suggests an increase in the number of apoptotic cells at this early time point. DAPI counterstain (blue). Scale bar $=100 \mu \mathrm{m}$.

levels of both nutrients and chondrogenic induction factors, and so differences in local matrix formation. If chondrogenic MSCs are less able than chondrocytes to function under nutritional constraints, then gradients would tend to exacerbate differences between constructs formed from these two cell types. To investigate this possibility, the goal of this study was to quantify and compare the local properties of chondrocyte- and MSC-laden agarose constructs so as to better understand the underlying mechanisms that currently limit the clinical application of MSC-based engineered cartilage.

To carry out this study, we evaluated spatial and temporal production of extracellular matrix, and measured the local (depth dependent) properties of constructs via microscopic mechanical analysis. Here we show that, consistent with previous findings (Erickson et al., 2009; Huang et al., 2009; Mauck et al., 2006), the bulk properties of freeswelling MSC-laden constructs (cultured with TGF- $\beta 3$ ) increase with time, but plateau at a level significantly lower than chondrocytes. Microscopic analysis of local properties illustrated several important points that were not fully appreciated with macroscopic testing. First, the properties of all free-swelling constructs (both MSCand chondrocyte-based) were depth-dependent, with the highest properties measured at the top surface of the construct (where maximal nutrient exchange would be expected). Most interestingly, comparing properties within this superficial region, we found that MSC-based constructs matched or exceeded that of chondrocytebased constructs. These data indicate that MSCs are in fact capable of producing mechanically robust tissue, but can do so only under these optimal conditions. A second important finding emerged when we reduced diffusional constraints (by limiting unstirred layers with orbital shaking). Under these dynamic conditions, bulk properties of MSC-laden construct increased substantially, with local analysis showing equivalent properties between both chondrocytes and MSCs in both the superficial and deep zones. However, within the central region of MSC-based constructs, properties remained significantly lower than that of chondrocyte-based constructs cultured identically. When cultured under dynamic conditions, chondrocytebased constructs achieved a high and nearly linear profile in mechanical properties through the depth, while markedly lower properties persisted in the centers of MSC-based constructs (Fig. 3). This observation was supported by both semi-quantitative analysis of proteoglycan deposition through the depth (Fig. 4), as well as regional analysis of biochemical constituents (Fig. 6). Despite the measured depth-dependent mechanical profiles of chondrocyteladen FS(+TGF- $\beta 3$ ) constructs, proteoglycan deposition assessed by Alcian blue staining appeared relatively uniform. It is not clear whether this represents limitations in the sensitivity of this assay (i.e., inability to discriminate between higher concentrations of proteoglycan) or whether it suggests the presence of additional matrix components or structural organization that is critical for mechanical function. Furthermore, low proteoglycan staining intensity was observed at the very periphery of both dynamically cultured MSC and chondrocyte-laden constructs. One possible explanation for this may be loss of proteoglycans due to agitation of the constructs. However, as GAG content in the media was not measured, additional factors such as altered regional GAG production due to shear fluid forces at the periphery cannot be ruled out, and future studies on this topic are warranted.

From the MSC data, it was clear that the distance from the free edge is a critical determinant of matrix formation 
(and hence functional properties), and that these gradients were at least in part governed by diffusion limits in this 3D system. Cells far from a nutrient supply may either fail to fully differentiate (lacking a sufficient supply of pro-chondrogenic factors), or be so starved for nutrients that they fail to form matrix even after the differentiation event has occurred. This is an important and not often discussed feature of MSC chondrogenesis. That is, not only must MSCs differentiate to achieve anabolic functionality (i.e., matrix production) matching chondrocytes, but they also must function in a constrained and nutrient poor environment - this being a hallmark of how a chondrocyte operates in native cartilage tissue (Mobasheri et al., 2005; Schipani et al., 2001). In one recent study, it was reported that nutrient availability can impact the growth of even chondrocyte-based constructs, where, below a certain nutrient threshold, viability was markedly compromised within the center of constructs (Heywood et al., 2006; Heywood et al., 2004). Based on this, we quantified MSC viability throughout the construct depth, as a function of time, presence of TGF- $\beta 3$, and culture condition (static versus dynamic). Remarkably, while chondrocytes had a relatively stable level of viability overall and in each region of the construct, MSC-based constructs showed dramatic decreases in viability from day 1 levels for all culture conditions and within all regions. While viability was poor overall without chondrogenic induction (i.e., without TGF- $\beta 3$ ), striking declines in viability were noted within the central regions of free-swelling constructs, even when cultured with TGF- $\beta 3$. Of further note, these decreases in viability and positive TUNEL staining were present as early as $21 \mathrm{~d}$ into culture, at a time where depth dependent strain profiles were already established. Follow up studies (not shown), demonstrated that these declines in viability, and initiation of apoptotic cascades, begin as early as one week into culture, well before appreciable matrix has been deposited.

One further interesting observation of this study was that not every MSC within the central regions of constructs underwent cell death. Even under the most demanding conditions (central and bottom regions of free swelling constructs), a minor population survived, underwent chondrogenesis and produced matrix that was increasingly functional with time. It is well-appreciated that marked heterogeneity in differentiation potential exists in adult stem cell populations (Halleux et al., 2001; Mareddy et al., 2007; Okamoto et al., 2002; Pittenger et al., 1999; Russell et al., 2010). This heterogeneity in differentiation potential may have translated to heterogeneity in survival under these challenging microenvironmental conditions. The MSC sub-population that remained viable in the center of the constructs may represent a fraction of cells uniquely suited to take on the chondrogenic phenotype, addressing both anabolic and metabolic demands of tissue formation and in vivo function. For effective clinical repair or replacement of cartilage, a tissue analog must maintain its homeostatic state and appropriately remodel within the implant site. Cartilage is avascular and relies on diffusion for all nutrient exchange. If a portion of the MSC population is unable to survive in vitro in these constructs, where the nutritional gradients produced are created solely from cell utilization, the effects will likely be exacerbated when exposed to the low nutrient, low oxygen conditions of the synovial joint. Thus, identification of this subpopulation may be a critical step in furthering our goal of achieving a viable cell population throughout the construct, and improving chondrogenesis for in vitro and in vivo application.

Overall, this work demonstrates that MSCs are capable of creating robust and mechanically functional extracellular matrix that is comparable to chondrocytes in $3 \mathrm{D}$ culture. However, our findings also show that MSCs can only function in this manner in regions with ample nutrient supply. Although dynamic culture increased the mechanical properties of MSC-laden constructs on a macroscopic level, the marked decrease in mechanical properties through the depth revealed that persistent differences remain between the two cell types. The observed decreases in cell viability provide some explanation for the mechanical deficits we measured, and point to a new frame of reference by which to judge the efficiency of chondrogenic induction.

On a molecular level, anabolic function by MSCs is robust, while their ability to function and persevere in a constrained environment appears to be lacking. As nutrients are consumed from the edge of the construct to the center, a condition of low glucose, low oxygen, and absence of chondrogenic factors would likely be present. Chondrocytes are well suited to operate in this context, with robust pathways (including hypoxia inducible factor -1alpha (Schipani et al., 2001) and glucose transporters (Mobasheri et al., 2005)) that are tuned for operation in this native state of duress. While the prochondrogenic effects of low oxygen tension have been noted (Adesida et al., 2012), MSCs within the center of the constructs would likely experience both low oxygen and low nutrient conditions, the combinatorial effect of which has been shown to cause marked cell death in this cell type (Potier et al., 2007).

Differences in nutrient consumption and waste production rates between chondrocytes and MSCs may in fact be creating such gradients, providing drastically different microenvironments within individual constructs. If MSCs utilize vital resources in a differential manner compared to chondrocytes, particularly if they have higher anabolic activity as it appears they may at the periphery, conditions in the center of constructs would be further exacerbated. If nutrient consumption at the periphery could be attenuated slightly, or physical conduits (channels) were provided to improve media access to the center (Bian et al., 2009; Buckley et al., 2009), the health of the MSC population in the center of the constructs might be preserved at early time points. That some MSCs do survive and thrive under these conditions, however, speaks to the overall heterogeneity of these stem cell populations, and suggests that proper sorting of cells, based on anabolic and metabolic chondrogenic efficiency, may yield improved in vivo tissue regeneration through an optimized cell population. Taken together, these data better identify crucial underlying mechanisms that have limited the clinical potential of chondrogenic MSCs, and provide new strategies for bringing stem cell-based cartilage tissue replacements to the clinic. 


\section{Acknowledgements}

This work was supported by the National Institutes of Health (R01 EB008722) and the Human Frontiers in Science Foundation. The authors would like to thank Drs. Alice Huang and Isaac Erickson for their help and expertise. We wish to confirm that there are no known conflicts of interest associated with this publication and there has been no significant financial support for this work that could have influenced its outcome.

\section{References}

Adesida AB, Mulet-Sierra A, Jomha NM (2012) Hypoxia mediated isolation and expansion enhances the chondrogenic capacity of bone marrow mesenchymal stromal cells. Stem Cell Res Ther 3: 9.

Ateshian GA, Hung CT (2005) Patellofemoral joint biomechanics and tissue engineering. Clin Orthop Relat Res 436: 81-90.

Bian L, Angione SL, Ng KW, Lima EG, Williams DY, Mao DQ, Ateshian GA, Hung CT (2009) Influence of decreasing nutrient path length on the development of engineered cartilage. Osteoarthritis Cartilage 17: 677-685.

Boeuf S, Steck E, Pelttari K, Hennig T, Buneb A, Benz K, Witte D, Sultmann H, Poustka A, Richter W (2008) Subtractive gene expression profiling of articular cartilage and mesenchymal stem cells: serpins as cartilage-relevant differentiation markers. Osteoarthritis Cartilage 16: 48-60.

Buckley CT, Meyer EG, Kelly DJ (2012) The influence of construct scale on the composition and functional properties of cartilaginous tissues engineered using bone marrow-derived mesenchymal stem cells. Tissue Eng Part A 18: 382-396.

Buckley CT, Thorpe SD, Kelly DJ (2009) Engineering of large cartilaginous tissues through the use of microchanneled hydrogels and rotational culture. Tissue Eng Part A 15: 3213-3220.

Byers BA, Mauck RL, Chiang IE, Tuan RS (2008) Transient exposure to transforming growth factor beta 3 under serum-free conditions enhances the biomechanical and biochemical maturation of tissue-engineered cartilage. Tissue Eng Part A 14: 1821-1834.

Chung C, Burdick JA (2008) Engineering cartilage tissue. Adv Drug Deliv Rev 60: 243-262.

Dolgin E (2011) Taking tissue engineering to heart. Nat Med 17: 1032-1035.

Erickson IE, Huang AH, Chung C, Li RT, Burdick JA, Mauck RL (2009) Differential maturation and structurefunction relationships in mesenchymal stem cell- and chondrocyte-seeded hydrogels. Tissue Eng Part A 15: 1041-1052.

Farndale RW, Buttle DJ, Barrett AJ (1986) Improved quantitation and discrimination of sulphated glycosaminoglycans by use of dimethylmethylene blue. Biochim Biophys Acta 883: 173-177.

Friedenstein AJ, Chailakhjan RK, Lalykina KS (1970) The development of fibroblast colonies in monolayer cultures of guinea-pig bone marrow and spleen cells. Cell Tissue Kinet 3: 393-403.
Halleux C, Sottile V, Gasser JA, Seuwen K (2001) Multi-lineage potential of human mesenchymal stem cells following clonal expansion. J Musculoskelet Neuronal Interact 2: 71-76.

Heywood HK, Lee DA (2008) Monolayer expansion induces an oxidative metabolism and ROS in chondrocytes. Biochem Biophys Res Commun 373: 224-229.

Heywood HK, Sembi PK, Lee DA, Bader DL (2004) Cellular utilization determines viability and matrix distribution profiles in chondrocyte-seeded alginate constructs. Tissue Eng 10: 1467-1479.

Heywood HK, Bader DL, Lee DA (2006) Glucose concentration and medium volume influence cell viability and glycosaminoglycan synthesis in chondrocyte-seeded alginate constructs. Tissue Eng 12: 3487-3496.

Huang AH, Stein A, Tuan RS, Mauck RL (2009) Transient exposure to transforming growth factor beta 3 improves the mechanical properties of mesenchymal stem cell-laden cartilage constructs in a density-dependent manner. Tissue Eng Part A 15: 3461-3472.

Huang AH, Farrell MJ, Kim M, Mauck RL (2010a) Long-term dynamic loading improves the mechanical properties of chondrogenic mesenchymal stem cell-laden hydrogel. Eur Cell Mater 19: 72-85.

Huang AH, Farrell MJ, Mauck RL (2010b) Mechanics and mechanobiology of mesenchymal stem cell-based engineered cartilage. J Biomech 43: 128-136.

Huang AH, Stein A, Mauck RL (2010c) Evaluation of the complex transcriptional topography of mesenchymal stem cell chondrogenesis for cartilage tissue engineering. Tissue Eng Part A 16: 2699-2708.

Hung CT, Mauck RL, Wang CC, Lima EG, Ateshian GA (2004) A paradigm for functional tissue engineering of articular cartilage via applied physiologic deformational loading. Ann Biomed Eng 32: 35-49.

Johnstone B, Hering TM, Caplan AI, Goldberg VM, Yoo JU (1998) In vitro chondrogenesis of bone marrowderived mesenchymal progenitor cells. Exp Cell Res 238: 265-272.

Kelly TA, Ng KW, Wang CC, Ateshian GA, Hung CT (2006) Spatial and temporal development of chondrocyteseeded agarose constructs in free-swelling and dynamically loaded cultures. J Biomech 39: 1489-1497.

Klein TJ, Sah RL (2007) Modulation of depthdependent properties in tissue-engineered cartilage with a semi-permeable membrane and perfusion: a continuum model of matrix metabolism and transport. Biomech Model Mechanobiol 6: 21-32.

Klein TJ, Chaudhry M, Bae WC, Sah RL (2007) Depthdependent biomechanical and biochemical properties of fetal, newborn, and tissue-engineered articular cartilage. J Biomech 40: 182-190.

Knight MM, Lee DA, Bader DL (1998) The influence of elaborated pericellular matrix on the deformation of isolated articular chondrocytes cultured in agarose. Biochim Biophys Acta 1405: 67-77.

LaPrade RF, Bursch LS, Olson EJ, Havlas V, Carlson CS (2008) Histologic and immunohistochemical characteristics of failed articular cartilage resurfacing procedures for osteochondritis of the knee: a case series. Am J Sports Med 36: 360-368. 
Lima EG, Bian L, Ng KW, Mauck RL, Byers BA, Tuan RS, Ateshian GA, Hung CT (2007) The beneficial effect of delayed compressive loading on tissue-engineered cartilage constructs cultured with TGF-beta3. Osteoarthritis Cartilage 15: 1025-1033.

Macchiarini P, Jungebluth P, Go T, Asnaghi MA, Rees LE, Cogan TA, Dodson A, Martorell J, Bellini S, Parnigotto PP, Dickinson SC, Hollander AP, Mantero S, Conconi MT, Birchall MA (2008) Clinical transplantation of a tissueengineered airway. Lancet 372: 2023-2030.

Mareddy S, Crawford R, Brooke G, Xiao Y (2007) Clonal isolation and characterization of bone marrow stromal cells from patients with osteoarthritis. Tissue Eng 13: 819-829.

Mauck RL, Soltz MA, Wang CC, Wong DD, Chao PH, Valhmu WB, Hung CT, Ateshian GA (2000) Functional tissue engineering of articular cartilage through dynamic loading of chondrocyte-seeded agarose gels. J Biomech Eng 122: 252-260.

Mauck RL, Wang CC, Oswald ES, Ateshian GA, Hung CT (2003) The role of cell seeding density and nutrient supply for articular cartilage tissue engineering with deformational loading. Osteoarthritis Cartilage 11: 879-890.

Mauck RL, Yuan X, Tuan RS (2006) Chondrogenic differentiation and functional maturation of bovine mesenchymal stem cells in long-term agarose culture. Osteoarthritis Cartilage 14: 179-189.

Melrose J, Smith S, Ghosh P (2004) Histology and immunology studies in cartilage. Methods Mol Med 101: 39-63.

Meyer EG, Buckley CT, Steward AJ, Kelly DJ (2011) The effect of cyclic hydrostatic pressure on the functional development of cartilaginous tissues engineered using bone marrow derived mesenchymal stem cells. J Mech Behav Biomed Mater 4: 1257-1265.

Mobasheri A, Richardson S, Mobasheri R, Shakibaei M, Hoyland JA (2005) Hypoxia inducible factor-1 and facilitative glucose transporters GLUT1 and GLUT3: putative molecular components of the oxygen and glucose sensing apparatus in articular chondrocytes. Histol Histopathol 20: 1327-1338.

Nehrer S, Spector M, Minas T (1999) Histologic analysis of tissue after failed cartilage repair procedures. Clin Orthop Relat Res 365: 149-162.

Okamoto T, Aoyama T, Nakayama T, Nakamata T, Hosaka T, Nishijo K, Nakamura T, Kiyono T, Toguchida $\mathrm{J}$ (2002) Clonal heterogeneity in differentiation potential of immortalized human mesenchymal stem cells. Biochem Biophys Res Commun 295: 354-361.

Pattappa G, Heywood HK, de Bruijn JD, Lee DA(2011) The metabolism of human mesenchymal stem cells during proliferation and differentiation. J Cell Physiol 226: 25622570 .

Pittenger MF, Mackay AM, Beck SC, Jaiswal RK, Douglas R, Mosca JD, Moorman MA, Simonetti DW, Craig S, Marshak DR (1999) Multilineage potential of adult human mesenchymal stem cells. Science 284: 143-147.

Potier E, Ferreira E, Meunier A, Sedel L, LogeartAvramoglou D, Petite H (2007) Prolonged hypoxia concomitant with serum deprivation induces massive human mesenchymal stem cell death. Tissue Eng 13: $1325-$ 1331.

Russell KC, Phinney DG, Lacey MR, Barrilleaux BL, Meyertholen KE, O’Connor KC (2010) In vitro highcapacity assay to quantify the clonal heterogeneity in trilineage potential of mesenchymal stem cells reveals a complex hierarchy of lineage commitment. Stem Cells 28: 788-798.

Schinagl RM, Gurskis D, Chen AC, Sah RL (1997) Depth-dependent confined compression modulus of fullthickness bovine articular cartilage. J Orthop Res 15: 499-506.

Schipani E, Ryan HE, Didrickson S, Kobayashi T, Knight M, Johnson RS (2001) Hypoxia in cartilage: HIF-1alpha is essential for chondrocyte growth arrest and survival. Genes Dev 15: 2865-2876.

Stegemann H, Stalder K (1967) Determination of hydroxyproline. Clin Chim Acta 18: 267-273.

Van Assche D, Staes F, Van Caspel D, Vanlauwe J, Bellemans J, Saris DB, Luyten FP (2010) Autologous chondrocyte implantation versus microfracture for knee cartilage injury: a prospective randomized trial, with 2-year follow-up. Knee Surg Sports Traumatol Arthrosc 18: 486495.

Vijayan S, Bentley G, Briggs T, Skinner J, Carrington R, Pollock R, Flanagan A (2010) Cartilage repair: A review of Stanmore experience in the treatment of osteochondral defects in the knee with various surgical techniques. Indian J Orthop 44: 238-245.

Wang CC, Hung CT, Mow VC (2001) An analysis of the effects of depth-dependent aggregate modulus on articular cartilage stress-relaxation behavior in compression. J Biomech 34: 75-84.

\section{Discussion with Reviewers}

Reviewer I: The engineered constructs are relatively small (in both their thickness and diameter) compared to clinically sized defects, yet significant gradients in matrix accumulation develop. Can the authors comment on the implications of their findings for engineering larger sized constructs? Do they believe the use of dynamic culture could be equally successful for engineering such tissues, or might further developments be required?

Authors: Scaling up in any tissue engineered construct is always a significant consideration that will need to be addressed. Here, our constructs started at $2.25 \mathrm{~mm}$ in thickness, which covers most, but certainly not all, relevant thicknesses of cartilage in the human body. In this work, dynamic culture did somewhat attenuate the gradients observed in these constructs, and would likely be beneficial for larger constructs as well. In addition to orbital shaking, other loading modalities, such as compression or sliding contact, may likewise improve fluid/nutrient exchange. Once constructs achieve a very large size, however, other more macroscopic methods (i.e., channels) might be required, as has been reported for chondrocyte and MSCseeded gels (Albro et al., 2011; Bian et al., 2009; Buckley et al., 2009). 
Reviewer I: Can the authors expand on how they might be able identify the "subpopulation" of MSCs that can thrive in challenging nutrient conditions.

Authors: We are currently working to expose heterogeneous parent MSC populations and subpopulations to defined challenging conditions to determine how these cells respond to stress. If a hardy and efficient subpopulation can be isolated, and if they can be expanded without losing this unique capacity, then a 'superior' construct might be formed that responds to the constraints of the culture environment and synovial joint in a fashion that is more similar to that of chondrocytes. We are actively engaged in such analyses, and this will be the subject of future work should it prove feasible.

Reviewer II: Would one expect limitation of oxygen diffusion in these studies? If so, hypoxia would seem to be a more chondrogenic permissive environment for tissue development?

Authors: These studies were performed in normal (21\% oxygen conditions). In general, low oxygen tension and hypoxic preconditioning have been reported to enhance chondrogenesis, and we would expect that consumption of this factor would decrease its availability at the center relative to the edge (Buckley et al., 2012). However, MSCs in the center of the construct are likely to experience a challenging environment that is not only hypoxic, but also low in nutrients, including glucose and TGF-beta, as well as high in metabolic waste. Hypoxic conditions favor anaerobic energy utilization, and therefore the presence of glucose and other nutrients might be beneficial (or necessary) in such an environment. Indeed, it has been reported that serum deprivation coupled with low oxygen can significantly compromise MSC health (Potier et al., 2007), and could contribute to the viability issues we see at the center of constructs.

\section{Additional Reference}

Albro MB, Banerjee RE, Li R, Oungoulian SR, Chen B, del Palomar AP, Hung CT, Ateshian GA (2011) Dynamic loading of immature epiphyseal cartilage pumps nutrients out of vascular canals. J Biomech 44: 1654-1659. 